\title{
Modelling influenza A H5N1 vaccination strategy scenarios in the household poultry sector in Egypt
}

\author{
Ihab EI Masry • Jolianne Rijks • Marisa Peyre • \\ Nick Taylor • Juan Lubroth • Yilma Jobre
}

Accepted: 1 July 2013 / Published online: 19 July 2013

(C) The Author(s) 2013. This article is published with open access at Springerlink.com

\begin{abstract}
Highly pathogenic avian influenza (AI) due to H5N1 virus was first reported in Egypt in February 2006; since then, the government has allowed avian influenza vaccination in poultry. The present study evaluated the impact of AI vaccination in terms of cumulative annual flock immunity (CAFI): the percentage of bird $\times$ weeks protected by immunity. This evaluation took account of the combined effects of vaccination coverage, vaccine efficacy (VE), and different characteristics of household poultry production on the effectiveness of the adopted vaccination strategy (VS),
\end{abstract}

\footnotetext{
I. El Masry $(\bowtie) \cdot$ Y. Jobre

Emergency Centre for Transboundary Animal Disease,

Food and Agriculture Organization of the United Nations (FAO), P.O. Box 2223, Cairo, Egypt

e-mail: ihab.elmasry@fao.org

Y. Jobre

e-mail: yilma.jobre@fao.org

J. Rijks

Dutch Wildlife Health Centre, Yalelaan 1, 3584CL Utrecht,

The Netherlands

e-mail: j.m.rijks@uu.nl

M. Peyre

Animal et Gestion Intégrée des Risques, Centre International de Recherche en Agriculture pour le Développement,

34398 Montpellier, France

e-mail: marisa.peyre@cirad.fr

N. Taylor

Veterinary Epidemiology and Economics Research Unit, Reading

University, School of Agriculture, Policy and Development,

Berkshire, UK

e-mail: n.m.taylor@reading.ac.uk

J. Lubroth

Food and Agriculture Organization of the United Nations (FAO),

Rome, Italy

e-mail: Juan.Lubroth@fao.org
}

and provided alternative options for improvement. The evaluation used a population and vaccination model that calculates the CAFI. Participatory approaches were employed in 21 villages to develop the vaccination and flock parameters required for the model. The adopted VS were compared in the model with three alternative VS scenarios in terms of the CAFI. Vaccination coverage varied among villages but was generally low (between 1 and $48 \%$; median $14 \%$ ). Under the adopted VS, the CAFI predicted for the villages ranged from 2 to $31 \%$. It was concluded that despite the enormous effort put into rural household poultry AI vaccination by the Egyptian government, village CAFI is unlikely to be maintained at the levels required to significantly reduce the virus load and restrict transmission. In HPAI-endemic countries that consider $\mathrm{AI}$ vaccination as one of the disease control options, the high cost of mass AI vaccination campaigns and their achievable benefits must be compared with other available control measures, which may include targeted vaccination. Achievable vaccination coverage, $\mathrm{VE}$ and the different characteristics of commercial and household (village) poultry production are key parameters determining the feasibility and costeffectiveness of different AI vaccination strategies.

Keywords Avian influenza · HPAI H5N1 - Egypt · Vaccination coverage $\cdot$ Flock immunity $\cdot$ Vaccination strategy

\section{Introduction}

Highly pathogenic avian influenza (HPAI) H5N1 virus was detected in the Middle East (Egypt, Iraq, Iran, Israel, Jordan, Kuwait, Palestinian Territories) between November 2005 and March 2006. The virus was detected in Sudan and Djibouti in April 2006 and in Saudi Arabia in March 2007 (Williams and Peterson 2009). Of these countries, Egypt has been most severely affected. Poultry production in Egypt is present in many diverse forms, from large- and small-scale 
commercial sectors, to 'traditional' household poultry (HP) keeping.

HPAI due to the H5N1 subtype was first reported in poultry in Egypt in February 2006 (World Organization for Animal Health (OIE), 2006), and the first human case was reported in March 2006. The government allowed avian influenza (AI) vaccination in poultry shortly thereafter. During the period covered by this study (July 2007 to April 2009), AI vaccination was the main tool used to control HPAI in HP in Egypt. Some countries such as India and Bangladesh refrained from using vaccination as part of their control and prevention strategy (Food and Agriculture Organization of the United Nations (FAO), 2011). The General Organization for Veterinary Services (GOVS) is responsible for AI vaccination campaigns in Egypt. During the study period, four bi-annual house-to-house mass vaccination campaigns were implemented. In total, 212.5 million doses of inactivated AI vaccines, mainly of H5N1 but also H5N2 viruses, were used. The objectives of these campaigns were to reduce the virus load and transmission.

The combined impact of the vaccination coverage (VC), vaccine efficacy (VE), and different types of HP production on overall vaccination effectiveness, in terms of protection of the population, were neither sufficiently studied nor well documented in Egypt. Proper understanding of these factors, however, provides an insight into probable flock immunity levels and thereby, a clue to the effectiveness of the vaccination strategy (VS) in terms of attaining the desired objectives.

Participatory approaches were employed to collect data to be fed into a "population and vaccination model" version 8.1 (FAO 2008-unpublished consultancy report), to calculate the cumulative annual flock immunity (CAFI): the percentage of bird $\times$ weeks protected by immunity.

The objective of the present study was to compare the current AI VS adopted for the HP sector in Egypt with three alternative strategies in terms of the CAFI, and recommend a suitable AI VS.

\section{Materials and methods}

The model

The 'population and vaccination model' was developed to estimate the impact of different vaccination strategies on CAFI. It simulates the dynamic poultry population of a village, updating the status of the population at weekly intervals. The model also simulates development of immunity in the population in response to vaccination. A key output of this model is CAFI, which is calculated for any time period and for all or a subset of the population (species, breed, age group) as follows:
CAFI $\frac{\sum \mathrm{nSib}}{\sum \mathrm{nTnb}}$

Where $\Sigma \mathrm{nSib}=$ the sum of successfully immunised bird $\times$ weeks (i.e., considered protected) within a given poultry population across $n$ weeks; $\Sigma n T n b=$ the sum of all present bird $\times$ weeks within the same poultry population across $n$ weeks (irrespective of their immunity status). Because the model recalculates both the total number of birds present and birds immune on a weekly basis, the units in the CAFI equation are bird $\times$ weeks. In this paper, the CAFI result is presented as a percentage, and the period of calculation is 52 weeks.

In mixed-village flocks (with multiple species/breeds presenting different production and vaccination response characteristics), the flock was modelled using separate models for each sub-population. The overall CAFI is an aggregate of the CAFIs of each sub-population weighted by the relative proportion of each sub-population in the total village flock.

Study population and data collection

The characteristics of HP production in different localities was studied based on data collected in 34 randomly selected (out of 413) villages in Dakahlia (DK) governorate. Additionally, 12 villages were purposively selected from Minya, Asyut and Qena governorates. DK governorate is located in Lower Egypt where several village typologies are present. The remaining governorates are from Upper Egypt (UE) where there is more evident heterogeneity in village typology.

A group of public veterinarians, having received training on participatory epidemiology, collected raw data that were analyzed and used as model inputs, providing village flock production (Table 1) and vaccination parameters. Vaccination parameters included frequency and timing of vaccination campaigns per year, $\mathrm{VC}$, number of recommended vaccinations per species per annum, number of vaccinations implemented per species per annum, VE and the duration of immunity (DOI) following the initial and booster shots.

The process of data collection involved interviews with village key informants, a transect walk involving 15-20 house visits with individual interviews, observation of household birds and finally, group meetings with key informants and producers, where preliminary findings were verified and data gaps were filled in. Of the 46 studied villages, due to data quality concerns, information collected from only 21 villages (14 in DK and 7 in UE) were used in the model simulations. 


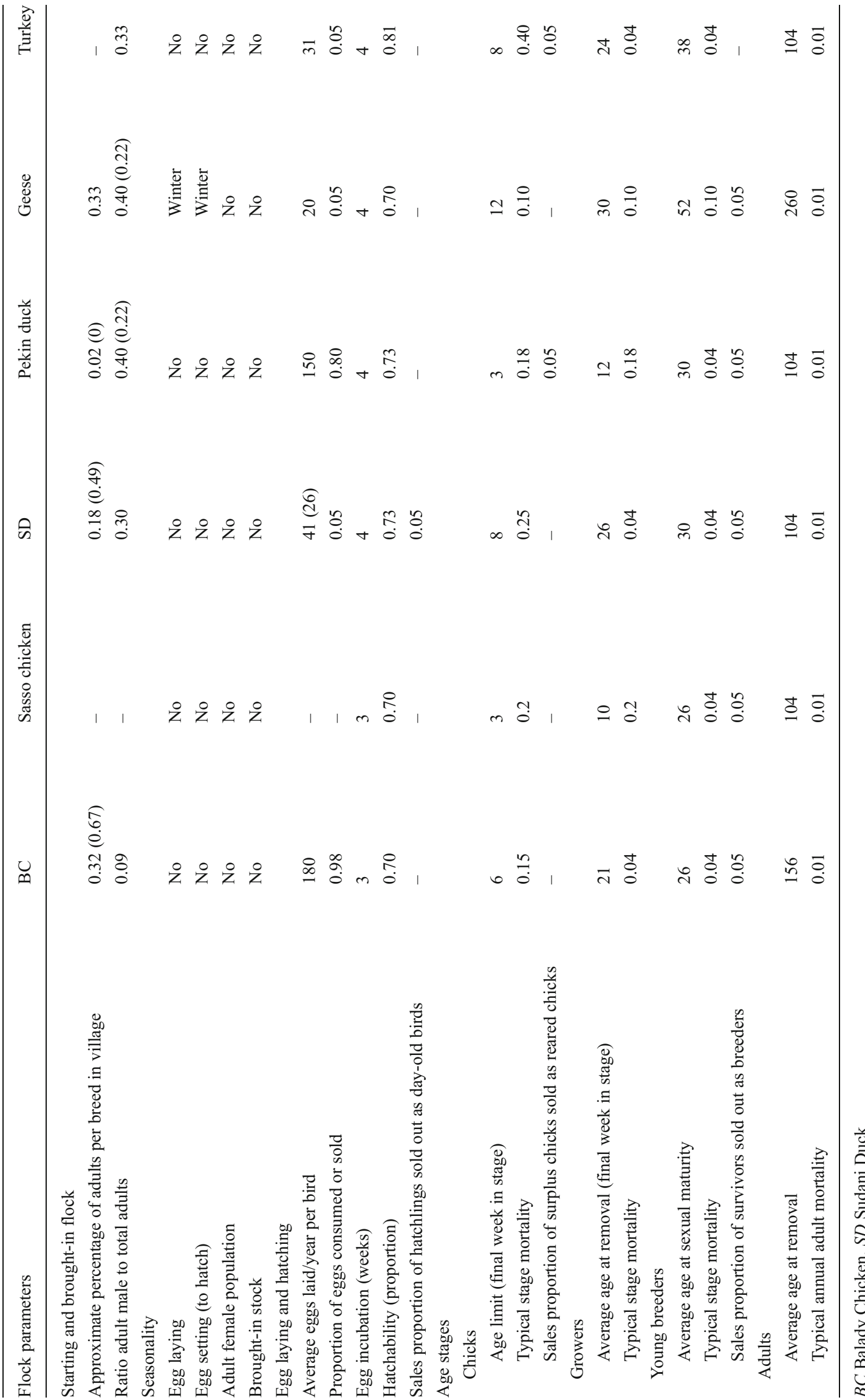




\section{Estimation of VC}

$\mathrm{VC}$ was estimated using the following formula:

$V C=\frac{\text { number of birds vaccinated during the latest campaign }}{\text { model estimate of poultry number in the village during the same period }}$

The relationship between village size and VC was statistically analyzed using Pearson's correlation coefficient $(r)$.

Modelling different vaccination strategies

The DOI was a variable vaccination parameter considered in the model. As prescribed by vaccine manufacturers, the value used as DOI for chicken and turkey was 24 weeks. For duck and geese, however, the DOI is either 4 or 16 weeks depending up on the absence or presence of a booster dose. The fixed vaccination parameters were as follows: (1) two vaccination campaigns per year with starting times set at week 1 and week 27, respectively; (2) the duration of the campaign lasting 1 week and (3) $80 \%$ VE. The VE level used in the model is the minimal required protection level (i.e., $80 \%$ ) as determined by the Central Laboratory for Evaluation of Veterinary Biologics for the registration of AI vaccines in Egypt. The vaccination parameters and the village flock production parameters were fed into the model and four VS scenarios (Table 2) were simulated.

\section{Results}

Poultry flock composition and dynamics

Six major categories of species and breeds with distinct production parameters (Table 1) were found in village flocks. These were indigenous (Balady) chicken (BC); Sasso and other hybrid chickens; Sudani ducks (SD) and Pekin and other meat ducks, geese and turkey. In general, BC was the largest category present in villages, and sometimes homebred but generally bought from outside sources including

Table 2 Parameters of the modelled four VS scenarios

\begin{tabular}{|c|c|c|c|c|}
\hline & Scenario 1 & Scenario 2 & Scenario 3 & Scenario 4 \\
\hline $\begin{array}{l}\text { Number of } \\
\text { campaigns } \\
\text { per year }\end{array}$ & 2 & 2 & 2 & 2 \\
\hline VC per campaign & Real VC & Real VC & $70 \%$ & $70 \%$ \\
\hline $\begin{array}{l}\text { Number of dose } \\
\text { per campaign }\end{array}$ & 1 & 1 & 1 & 1 \\
\hline $\begin{array}{l}\text { booster vaccination } \\
\text { for waterfowl }\end{array}$ & No & No & Yes & Yes \\
\hline $\begin{array}{l}\text { VC of brought-in } \\
\text { stocks }\end{array}$ & $0 \%$ & $80 \%$ & $0 \%$ & $80 \%$ \\
\hline
\end{tabular}

hatcheries, peddlers, markets or farms. The age at which the BCs were bought by households ranged from 1-30 days. These birds were raised either for meat and/or egg production. Conversely, home breeding was the main source of SD and geese. Sasso and other hybrid chickens, Pekin and other meat ducks were often brought to households at age of 121 days from the fore-mentioned sources of $\mathrm{BC}$, these types of chicken and ducks are kept for short duration as growers, and slaughtered at 2.5-3 months old.

\section{Estimation of VC}

The estimated VC showed great variation between villages. It ranged between $1-48 \%(n=21$, median $14 \%)$. Village size was negatively correlated with VC (Fig. $1 ; n=21, r=-0.49$, $P=0.026)$.

\section{CAFI}

The CAFI predictions for the 21 villages under the four VS scenarios are presented in Table 3.

\section{Flock characteristics and CAFI}

Statistical analysis revealed that CAFI is negatively correlated with standing flock size, and the proportion of waterfowl in the village. When $\mathrm{VC}$ is fixed at $70 \%$, a booster dose for waterfowl is given and when none of the brought-in stock are immune, the predicted CAFI decreases significantly as the average number of birds kept per poultry household increased ( $n=21, r=-0.640, P=0.002)$ and as the proportion of waterfowl increased $(n=21, \quad r=-0.524, \quad P=0.015)$ (Table 3).

\section{$\mathrm{VC}$ and $\mathrm{CAFI}$}

The CAFI for each village under scenario 1 has been plotted against the estimated VC in each village (Fig. 2). The model calculates the number of doses used under the different scenarios. The ratio between the numbers of doses in scenario 3 and the numbers of doses in scenario 1 was plotted against the estimated VC (Fig. 3), the resulting curve indicates for different levels of real VC the multiplying factor for the number of vaccine doses that would be required to evolve from scenarios 1 to 3 .

\section{Discussion}

The countries in which HPAI H5N1 virus is currently considered endemic include Bangladesh, China, Egypt, Indonesia, Vietnam as well as the Indian State of West Bengal (Hinrichs and Otte 2012). Waves of AI outbreaks may occur 


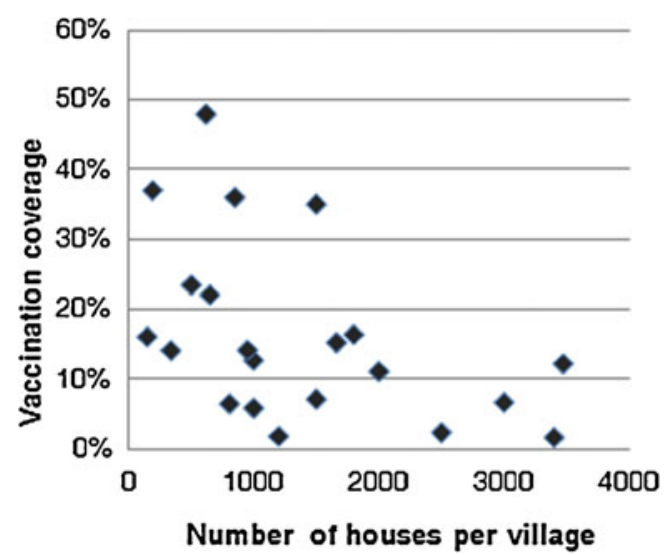

Fig. 1 Negative correlation between estimated village size and VC

in poultry populations in endemic areas whenever large parts of the population are left with a low level of immunity as a result of low VC (Magalhaes et al. 2006).

The present study represents an assessment of the interaction of VS with poultry production parameters that affect population dynamics on the CAFI. The level of village CAFI depended on the VE and VC achieved. The latter varied between villages but was generally low. The $80 \%$ VE level is in agreement with results obtained by Terregino et al. (2010), who confirmed that conventional vaccine is sufficient to prevent infection in the majority of birds challenged with a contemporary H5N1 Egyptian strain, and results obtained by Lebdah and Shahin (2010), who found an 80-92 \% protection level in chickens vaccinated with either $\mathrm{H} 5 \mathrm{~N} 1$ or H5N2 vaccines.

CAFI is also affected by the flock composition and production parameters. The six categories of poultry species and breeds distinguished at village level differed in terms of sources, numbers, turnover and production goals. These differences influence the results achieved by the adopted VS. SD and geese are homebred so they can only be immunised by vaccination at household level, and in that sense, the adopted VS is appropriate for them. However, the modelled CAFI is questionable because the required booster shot for waterfowl is rarely given. Also, unlike the seasonal hatching of geese, hatching of Sudani ducklings is continuous which creates a steady increase in the proportion of unvaccinated ducks in the time between campaigns, with subsequent quick decline in the CAFI in the SD population.

For Pekin duck, the CAFI modelled under the adopted strategy was limited because the required booster shot was
Table 3 Predicted CAFI in 21 villages under the four VS scenarios

\begin{tabular}{|c|c|c|c|c|c|c|}
\hline \multirow{2}{*}{$\begin{array}{l}\text { Village } \\
\text { number }\end{array}$} & \multirow{2}{*}{$\begin{array}{l}\text { Average number } \\
\text { of poultry per } \\
\text { household }\end{array}$} & \multirow{2}{*}{$\begin{array}{l}\text { Percentage of } \\
\text { waterfowl }(\%)\end{array}$} & \multicolumn{4}{|c|}{ Predicted CAFI } \\
\hline & & & $\begin{array}{l}\text { Scenario } \\
1(\%)\end{array}$ & $\begin{array}{l}\text { Scenario } \\
2(\%)\end{array}$ & $\begin{array}{l}\text { Scenario } \\
3(\%)\end{array}$ & $\begin{array}{l}\text { Scenario } \\
4(\%)\end{array}$ \\
\hline 1 & 23 & 41 & 18 & 38 & 49 & 66 \\
\hline 2 & 47 & 45 & 22 & 51 & 37 & 63 \\
\hline 3 & 46 & 50 & 16 & 38 & 37 & 62 \\
\hline 4 & 27 & 33 & 11 & 44 & 39 & 67 \\
\hline 5 & 38 & 31 & 31 & 64 & 42 & 72 \\
\hline 6 & 48 & 29 & 15 & 50 & 36 & 67 \\
\hline 7 & 60 & 35 & 5 & 42 & 37 & 68 \\
\hline 8 & 56 & 43 & 21 & 57 & 31 & 70 \\
\hline 9 & 14 & 35 & 17 & 40 & 49 & 70 \\
\hline 10 & 67 & 29 & 7 & 23 & 41 & 61 \\
\hline 11 & 18 & 27 & 14 & 48 & 40 & 71 \\
\hline 12 & 29 & 26 & 2 & 37 & 39 & 66 \\
\hline 13 & 37 & 58 & 21 & 45 & 36 & 62 \\
\hline 14 & 30 & 62 & 5 & 33 & 35 & 66 \\
\hline 15 & 17 & 12 & 17 & 54 & 47 & 75 \\
\hline 16 & 105 & 52 & 10 & 44 & 33 & 67 \\
\hline 17 & 48 & 66 & 11 & 41 & 44 & 84 \\
\hline 18 & 14 & 18 & 4 & 30 & 52 & 70 \\
\hline 19 & 25 & 9 & 8 & 51 & 46 & 73 \\
\hline 20 & 34 & 9 & 2 & 23 & 46 & 62 \\
\hline 21 & 43 & 39 & 11 & 43 & 41 & 69 \\
\hline Median & & & 11 & 41 & 40 & 67 \\
\hline
\end{tabular}




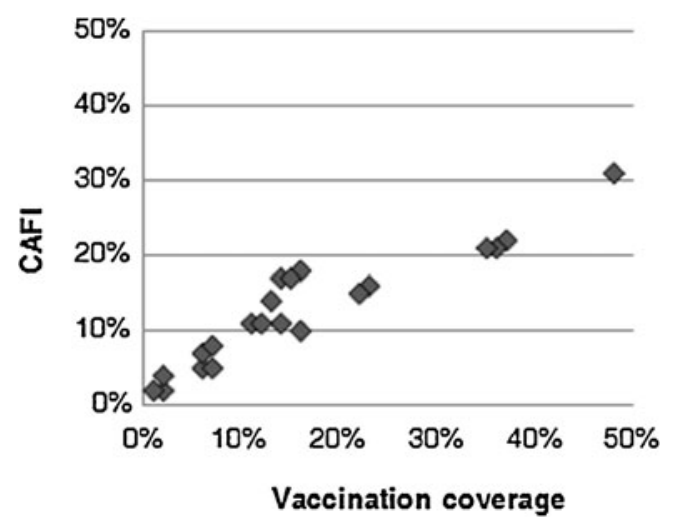

Fig. 2 Predicted CAFI in 21 villages under real VC (scenario 1)

not given and the short cycle (4-6 weeks) results in rapid removal of vaccinated Pekin ducks (to be replaced by unvaccinated ones). For BC, the level of CAFI achieved was largely dependent on the reason for keeping them. When the focus was solely on egg production, hens were kept for years with few new introductions. In such flocks, high levels of CAFI could be maintained with the adopted VS if VC was good. In contrast, when BCs were mainly kept as growers, turnover was greater and introductions occurred throughout the year, and levels of CAFI declined under the adopted VS, unless brought-in stock was also vaccinated.

Possible reasons for the negative correlation between standing flock size and CAFI in scenario 3 include that keeping high numbers of poultry requires more stock to be brought in (not immune in this scenario), while the negative correlation between proportion of waterfowl and CAFI can be attributed to the fact that DOI in waterfowl is shorter than the campaign interval (especially if booster shots are not delivered).

AI vaccination in free-range poultry flocks is usually implemented as periodic campaigns and new hatched chicks are generally not vaccinated between vaccination campaigns (Lesnoff et al. 2009). The high and recurrent costs, technical

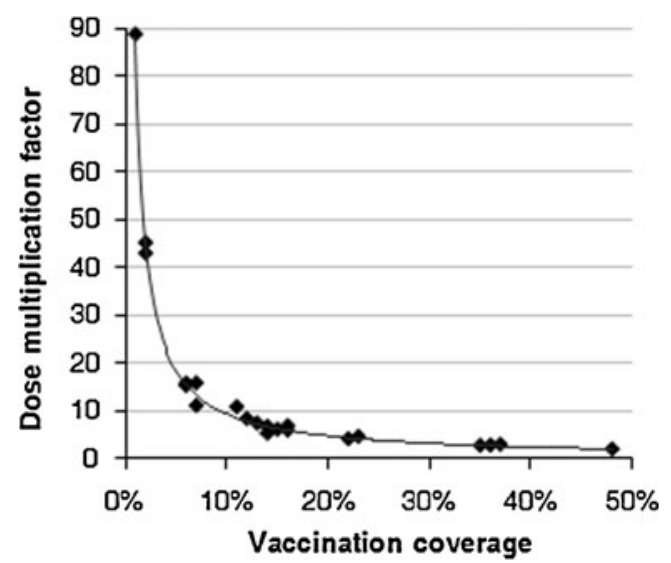

Fig. 3 The multiplying factor for the number of vaccine doses required to evolve from scenario 1 to scenario 3 difficulties, and epidemiological drawbacks of large-scale, open-ended and blanket vaccination programmes call for careful targeting of vaccination (Hinrichs et al. 2010). This study demonstrated the key beneficial influence on CAFI that may be achieved by vaccination of brought-in birds at source. In Egypt, it is common for village households to purchase birds at 3 or 4 weeks old that are reared at nursery farms. Targeting vaccination efforts at these nursery farms, all year round, would improve CAFI of HP populations.

\section{Conclusion}

Few countries are in a position to support governmentfunded mass vaccination campaigns (Food and Agriculture Organization of the United Nations 2011). The strategy of intensive countrywide vaccination as used in Vietnam was not implemented in Egypt and other biosecurity measures are not yet fully implemented (Kim et al. 2010). Nevertheless, and particularly, in HPAI-endemic countries, AI vaccination remains one of the main disease control options. This study highlights the importance of understanding key parameters such as VE, VC and campaign planning based on the different characteristics of the village poultry production in order to design and implement suitable AI vaccination strategies. The modelling outputs highlighted the difficulties in maintaining good flock immunity in HP with two vaccination rounds per year (Desvaux et al. 2012). The modelling approach may have a potential for extrapolation and could serve as a basis for similar studies on other major poultry diseases. Despite the enormous effort put into rural household poultry AI vaccination by the Egyptian government, village CAFI is unlikely to be maintained at the levels required to significantly reduce the virus load and restrict transmission. Reducing HPAI H5N1 viral load and transmission requires maintenance of high levels of flock immunity. This will require massive additional financial means, and it is questionable if it can be logistically feasible. The adopted AI VS of HP in Egypt has possibly become a net negative risk factor in the spread of the infection (Peyre et al. 2009). This is due to the fact that the positive impact in terms of raised CAFI is minimal while at the same time, the risk of transmitting HPAI among villages by the vaccination teams is a distinct possibility. AI vaccination absorbed 80 $90 \%$ of the budget for HPAI control put at the disposal of GOVS (Peyre et al. 2009). Targeted surveillance is needed in order to ensure that the protection achieved by mass vaccination is converted into a reduction in the likelihood of HPAI outbreaks occurring (Walker et al. 2010). The poor CAFI predicted by the model under the adopted VS suggests that more holistic packages of prevention and control measures should be explored. A package of surveillance and outbreak management measures, plus possibly targeted vaccination 
strategies (e.g., vaccination of birds at nursery farms) could be more effective. Before deciding on the continued use of vaccination as a tool for controlling HPAI, the outcome and the high cost of the vaccination campaigns must be compared with other available control measures in terms of costeffectiveness and feasibility.

Acknowledgements The authors would like to express their gratitude to the staff of the veterinary services; Dr. Rob de Rooij (former ECTAD Team Leader), Mr. Toni Ettel (ECTAD/EGY Programs Operation Officer) and Dr. Tony Wilsmore and Dr. Paolo Pagani (FAO Consultants) for their support during the field study. This work was part of the FAO project (OSRO/EGY/701/USA) funded by the United States Agency for International Development (USAID), for which the authors are grateful.

Open Access This article is distributed under the terms of the Creative Commons Attribution License which permits any use, distribution, and reproduction in any medium, provided the original author(s) and the source are credited.

\section{References}

Desvaux, S., Grosbois, V., Pham, T.T., Dao, D.T., Nguyen, T., Fenwick, S., Roger, F., Ellis, T. and Peyre,M., 2012. Evaluation of the vaccination efficacy against $\mathrm{H} 5 \mathrm{~N} 1$ in domestic poultry in the Red River Delta in Vietnam. Epidemiology and Infection.

FAO (Food and Agriculture Organization of the United Nations), 2011. Approaches to controlling, preventing and eliminating H5N1 Highly Pathogenic Avian Influenza in endemic countries. Animal Production and Health Paper No. 171. Food and Agriculture Organization of the United Nations, Rome, Italy.

Hinrichs, J. and Otte, J., 2012. Large-scale vaccination for the control of avian influenza: epidemiological and financial implications. In Zilberman D, Otte J, Roland-Holdst, Pfeiffer E (ed) Health and Animal Agriculture in Developing Countries, New York: Springer. 36:207-231.

Hinrichs, J., Otte, J. and Rushton, J., 2010. Technical, epidemiological and financial implications of large-scale national vaccination campaigns to control HPAI H5N1. CAB Reviews: Perspectives in
Agriculture, Veterinary Science, Nutrition and Natural Resources Vol. 5 No. 021 pp. 1-20.

Kim, J. K., Kayali, G., Walker, D., Forrest, H. L., Ellebedy, A. H., Griffin, Y. S., Rubrum, A., Bahgat, M. M., Kutkat, M. A., Ali, A., Aldridge, J. R., Negovetich, N. J., Krauss, S., Webby, R. J. and Webster, R. G., 2010. Puzzling inefficiency of H5N1 influenza vaccines in Egyptian poultry. Proceedings of the National Academy of Sciences of the United States of America, 15 June 2010, vol. 107, no. 24, pp. 11044-11049.

Lebdah, M. A. and Shahin, A. M., 2010. Evaluation of avian influenza vaccines used in broiler flocks in Egypt. Journal of American Science, 6(12).

Lesnoff, M., Peyre, M., Duarte, P. C., Renard, J. F. and Mariner, J. C., 2009. A simple model for simulating immunity rate dynamics in a tropical free-range poultry population after avian influenza vaccination. Epidemiology and Infection, 137, pp 1405-1413.

Magalhaes, R., Pfeiffer, D., Wieland, B., Dung, D. and Otte, J., 2006. Commune-level simulation model of HPAI H5N1 poultry infection and control in Vietnam. Pro-Poor Livestock Policy Initiative, Research Report, FAO, Rome, Italy, 8 pp.

OIE (World Organization for Animal Health), 2006. World animal health information database, summary of immediate notifications and follow-ups.

Peyre, M., Samaha, H., Makonnen, Y.J., Saad, A., Abd-Elnabi, A., Galal, S., Ettel, T., Dauphin, G., Lubroth, J., Roger, F. and Domenech, J., 2009. Avian influenza vaccination in Egypt: limitations of the current strategy. Journal of Molecular and Genetic Medicine, 3(2):198204.

Terregino, C., Toffan, A., Cilloni, F., Monne, I., Bertoli, E., Castellanos, L., Amarin, N., Mancin, M. and Capua, I., 2010. Evaluation of the protection induced by avian influenza vaccines containing a 1994 Mexican H5N2 LPAI seed strain against a 2008 Egyptian H5N1 HPAI virus belonging to clade 2.2 .1 by means of serological and in vivo tests. Avian Pathology, 39(3):215-22.

Walker, P. G., Cauchemez, S., Métras, R., Dung, H., Pfeiffer D. and Ghani, A. C., 2010. A Bayesian approach to quantifying the effects of mass poultry vaccination upon the spatial and temporal dynamics of H5N1 in Northern Vietnam. PLOS Computational Biology, $19 ; 6(2)$.

Williams, R.A.J., and Peterson, T.A., 2009. Ecology and geography of avian influenza (HPAI H5N1) transmission in the Middle East and northeastern Africa. International Journal of Health Geographics, $8: 47$. 